\title{
Effect of Three-Dimensional Printed Personalized Moisture Chamber Spectacles on the Periocular Humidity
}

\author{
Chan Hee Moon, ${ }^{1,2}$ Jae Yong Kim, ${ }^{1}$ Myoung Joon Kim, ${ }^{1}$ Hungwon Tchah, \\ Byeong Gak Lim, ${ }^{3}$ and Jin Kwon Chung ${ }^{4}$ \\ ${ }^{1}$ Department of Ophthalmology, Asan Medical Center, University of Ulsan College of Medicine, Seoul, Republic of Korea \\ ${ }^{2}$ Department of Ophthalmology, Aerospace Medical Center, Republic of Korea Air Force, Cheongju, Republic of Korea \\ ${ }^{3}$ Goodeye Clinic, Incheon, Republic of Korea \\ ${ }^{4}$ Department of Ophthalmology, Soonchunhyang University College of Medicine, Soonchunhyang University Hospital, \\ Seoul, Republic of Korea
}

Correspondence should be addressed to Jin Kwon Chung; schcornea@schmc.ac.kr

Received 20 June 2016; Revised 2 September 2016; Accepted 5 September 2016

Academic Editor: Jesús Pintor

Copyright (C) 2016 Chan Hee Moon et al. This is an open access article distributed under the Creative Commons Attribution License, which permits unrestricted use, distribution, and reproduction in any medium, provided the original work is properly cited.

Purpose. To assess the effect of three-dimensional (3D) printed personalized moisture chamber spectacles (PMCS) on the periocular humidity. Methods. Facial computed tomography (CT) scanning was conducted on 10 normal subjects. PMCS was designed based on volume rendered CT images and produced using a 3D printer. Periocular humidity of PMCS and commercially available uniformed moisture chamber spectacles (UMCS) were measured for 30 minutes via microhydrometer. Results. The mean ambient humidity was $15.76 \pm 1.18 \%$. The mean periocular humidity was $52.14 \pm 3.00 \%$ in PMCS and $37.67 \pm 8.97 \%$ in UMCS. The difference was significant $(P<0.001)$. Additionally, PMCS always demonstrated lower humidity than dew points. Conclusion. PMCS made by $3 \mathrm{D}$ printer provides appropriate fitness for the semiclosed humid chamber. PMCS showed higher performance than UMCS. The wearing of PMCS would be an effective method to provide high enough periocular humidity in low humidity environment.

\section{Introduction}

Dry eye is a common ocular disease prompting millions of individuals to seek ophthalmological care [1]. Artificial tear supplements are the most common form of treatment for dry eye. Moisture chamber spectacles are not used habitually in the treatment of dry eye; however they can be considered as an adjunct therapy in the case of severe dry eye when patients are recalcitrant to other means of treatment or special environment in which frequent instillation of eyedrops is unapplicable.

Korb et al. reported that increased periocular humidity using modified swim goggles resulted in an increase in tear film lipid layer thickness and moderate to total relief of dry eye symptoms [2]. Tsubota et al. attached small moistened sponges to side panels of spectacles to increase the moisture level near the cornea. Moisture chamber spectacles showed
$12.9 \%$ increase of periocular humidity and all enrolled dry eye patients noticed symptomatic relief with significantly decreased use of artificial tear [3].

Unlike general spectacles, moisture chamber spectacles need to be personalized in shape and size of frame for best performance. The tear retention effectiveness of moisture chamber spectacles is determined by the extent of proper semiclosed chamber formation. The formation of an appropriate chamber depends on how well the spectacles fit the facial contour. There are several commercially available moisture chamber spectacles; however they are single size or minimally adjustable. Previous studies were also performed using spectacles with single or several size variations $[2,3]$.

Three-dimensional (3D) printing was invented in the 1980s and has shown marked growth in recent years. 3D printing has been advancing in customized product printing and has proved to be effective for medical applications [4-6]. 


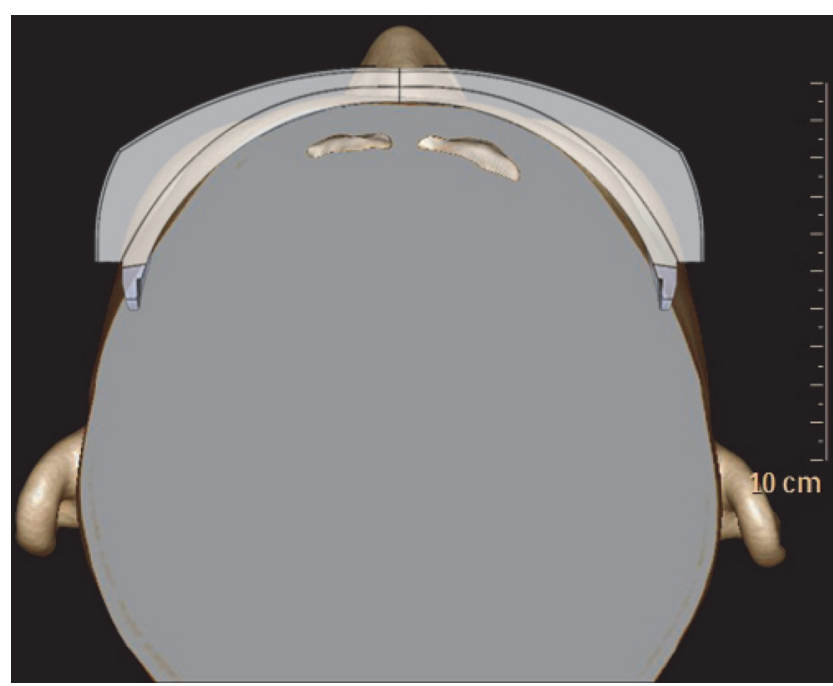

(a)

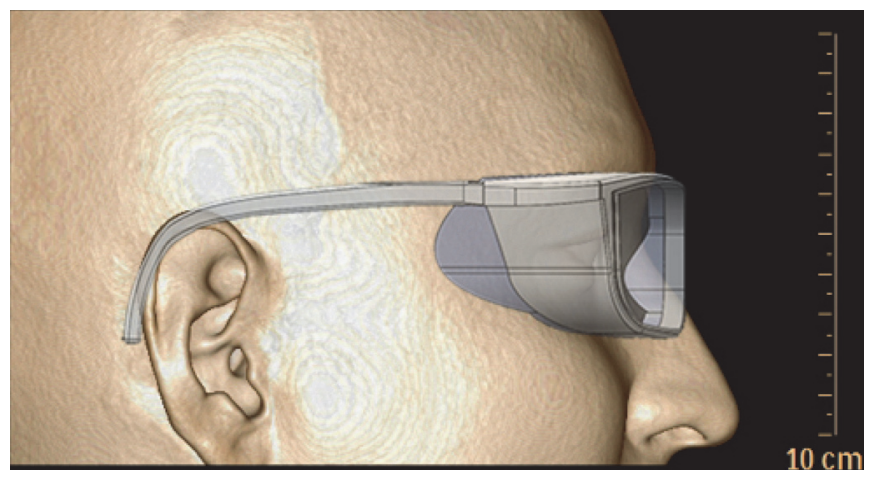

(c)

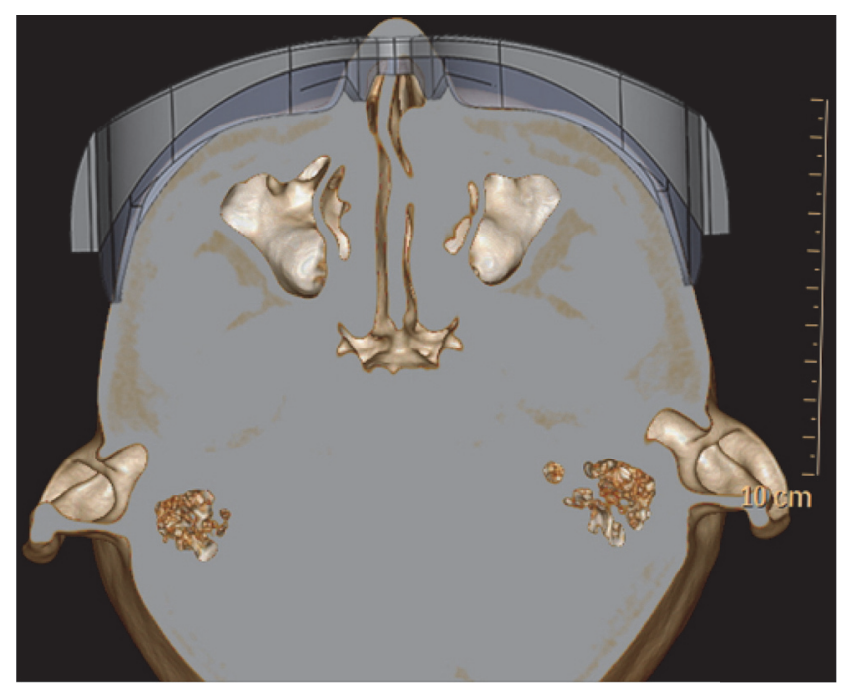

(b)

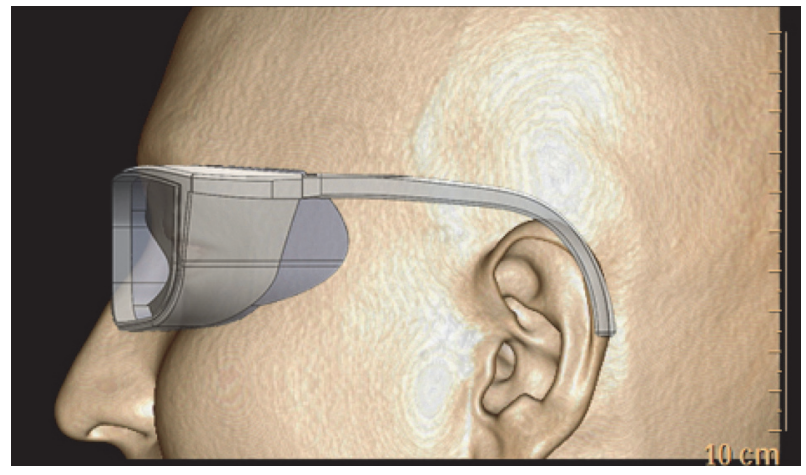

(d)

Figure 1: Personalization of moisture chamber spectacles using CT scan images. (a) Top, (b) bottom, (c) right side, and (d) left side.

Recently, 3D printing also has been highlighted in the field of ophthalmology [7-9]. One of the biggest advantages of 3D printing is to facilitate the production of personalized goods at affordable prices that are comparable with traditionally manufactured items.

In this study, personalized moisture chamber spectacles (PMCS) were produced using facial computed tomography (CT) scanning and 3D printing technology. The effect of PMCS on periocular humidity was evaluated and the clinical implication was discussed.

\section{Methods}

2.1. Subjects. This study was approved by the Institutional Review Board of research ethical committees of Aeromedical Center, Republic of Korea Air Force, and conformed to the tenets of the Declaration of Helsinki (ASMC-15-IRB-001). Ten normal subjects who have no history of evidence of ophthalmic disease were recruited.

2.2. Facial CT Scanning. Facial CT scanning for PMCS was conducted on the participants. Facial CT scanning was performed using a 64-channel CT scanner (Philips Brilliance
64; Philips Healthcare, Best, Netherlands). After conventional CT scan, 3D reconstruction was processed and facial contour was obtained using volume rendering tool, which was incorporated in the workstation (Figure 1).

2.3. Production of the PMCS. The spectacles were designed using computer-aided design (CAD) program (SolidWorks 2013; SolidWorks Corp., MA, USA). PMCS consisted of mainly 4 parts, that is, frame, side panel, moistened sponge block, and silicone chamber (Figure 2). All parts are personalized in size and shape, based on CT images. Frame and side panel were produced by 3D printer using polylactic acid material (Robox; CEL technology, North Somerset, UK). The width of the frame was set in bitemporal distance. The length of the temple was set in straight distance between frame and helix of ear for a little tight fitting. Posterior surface of silicone chamber was designed identically to the face contour. The side panels were designed to contain room for moistened sponge block. The inner side panel was made as many geographically perforated structures for providing humidity through passive evaporation from the moistened sponge. High absorptive melamine foam which consists of a formaldehyde-melamine-sodium bisulfite copolymer was 


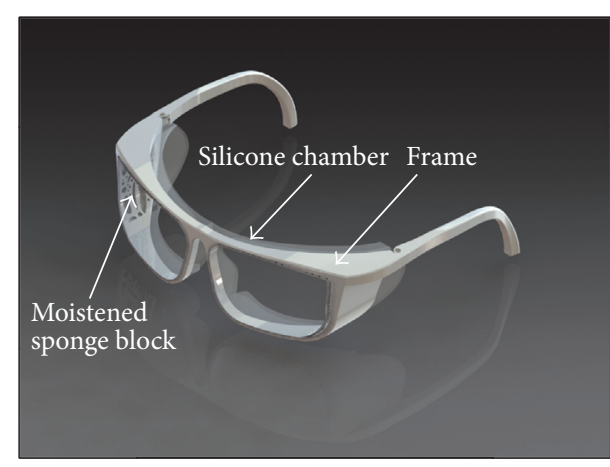

(a)

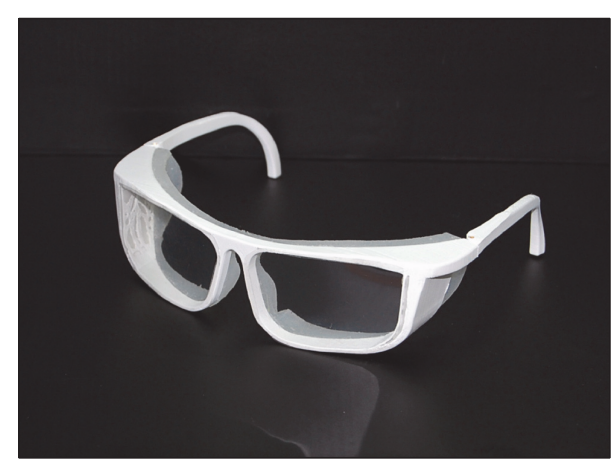

(b)

FIGURE 2: Rendering images and 3D printout of personalized moisture chamber spectacles. (a) Rendering images on CAD program. (b) 3D printed-out product.

used for moisture sponge block (Magic Eraser; The Procter \& Gamble Company, Ohio, USA). Sponge block had an average surface area of $778.5 \pm 13.6 \mathrm{~mm}^{2}$ and held $0.85 \mathrm{~g}$ of distilled water. Silicone part of moisture chamber was constructed on CAD and the mold was printed. Then silicone (Mold Master Ultra; Molkang, Paju, Korea) was infused into the mold and silicone chamber was formed.

2.4. Evaluation of Periocular Humidity and Temperature. The present study was designed as cross-over test. The subjects wore a sequence of different moisture chamber spectacles. Commercially available uniformed moisture chamber spectacles (UMCS) (Eyeeco, CA, USA) and PMCS were worn naturally for 30 minutes while periocular humidity and temperature were measured. UMCS were worn once again with gentle pressure on the spectacles frame in order to measure periocular humidity with nearly perfect sealing of moisture chamber. The order of the wearing spectacles was randomized. There was an interval of 30 minutes, prior to wearing other spectacles. The microhydrothermometer (AM2301; Aosong Electronics Co., Guangzhou, China) was attached to the backside of the spectacles lens (Figure 3). Left eyes were used for the analysis. The other identical hydrothermometer was used for ambient measurements. The humidity and temperature were measured every 30 seconds for 30 minutes. Room temperature and humidity were controlled at $22-23^{\circ} \mathrm{C}$ and $15-16 \%$ with thermoregulator and dehumidifier.

2.5. Calculation of Dew Points. The excessively high periocular humidity induces condensation on the spectacles lens and visual blur. The dew point is the temperature at which dew forms. A simple equation for the relationship between relative humidity and the dew point is as follows [10] $\left(t_{d}=\right.$ dew point, $t=$ temperature, and $\mathrm{RH}=$ relative humidity):

$$
t_{d}=t-\left(\frac{100-\mathrm{RH}}{5}\right)
$$

or

$$
\mathrm{RH}=100-5\left(t-t_{d}\right) .
$$

TABLE 1: Demographic characteristics.

\begin{tabular}{lc}
\hline Number of participants & 10 \\
Age (year) & $32 \pm 10.1$ \\
Sex (male/female) & $8 / 2$ \\
Interpupillary distance $(\mathrm{mm})$ & $62.4 \pm 5.5$ \\
Bitemporal distance $(\mathrm{mm})$ & $149.3 \pm 10.7$ \\
Temple length $(\mathrm{mm})$ & $81.5 \pm 8.0$ \\
\hline
\end{tabular}

The periocular humidity level which starts to cause condensation on the spectacles can be calculated by substituting periocular temperature for " $t$ " and ambient temperature for " $t_{d}$." The calculated RH and measured periocular humidity were compared.

2.6. Statistical Analysis. A repeated measures analysis of variance (rANOVA) and Bonferroni post hoc test were performed for comparisons among measurements. ShapiroWilk was used for normality test. Statistical analysis was conducted, using SPSS Statistics Version 21 (IBM Corporation, Somers, NY). All tests were two-tailed and $P<0.05$ was considered statistically significant.

\section{Results}

The mean age of participants was \pm years. Eight subjects were males and 2 were females. Facial characteristics including interpupillary distance, bitemporal distance, and temple length were described in Table 1.

3.1. Humidity. The hydrometer had an accuracy of $\pm 3 \% \mathrm{RH}$ and a repeatability of $\pm 1 \%$ RH. Measurements of periocular humidity over time were shown in Figure 4(a). The mean ambient humidity was $15.76 \pm 1.18 \%$. The mean periocular humidity was highest with pressurized wearing of UMCS (72.58 $\pm 4.42 \%)$, followed by natural wearing of PMCS $(52.15 \pm 3.00 \%)$ and natural wearing of UMCS $(37.67 \pm 8.96 \%)$. The differences of humidity measurements with various spectacles were significant $(P<0.001)$, (Figure 5(a)). 


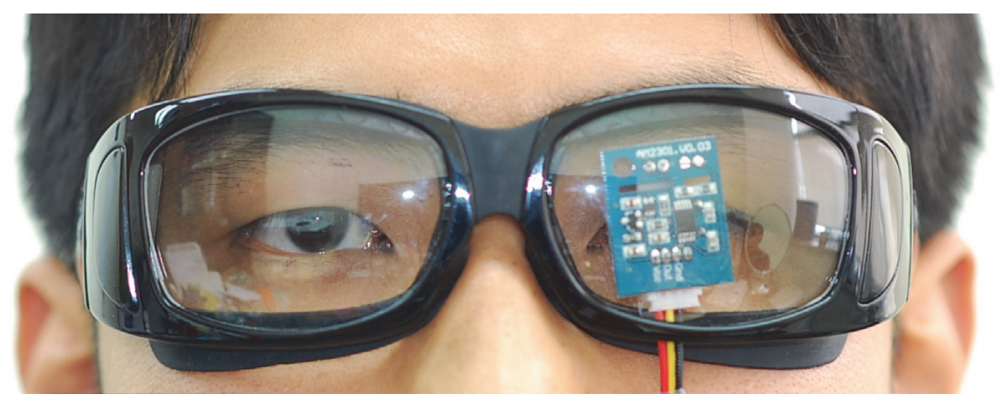

(a)

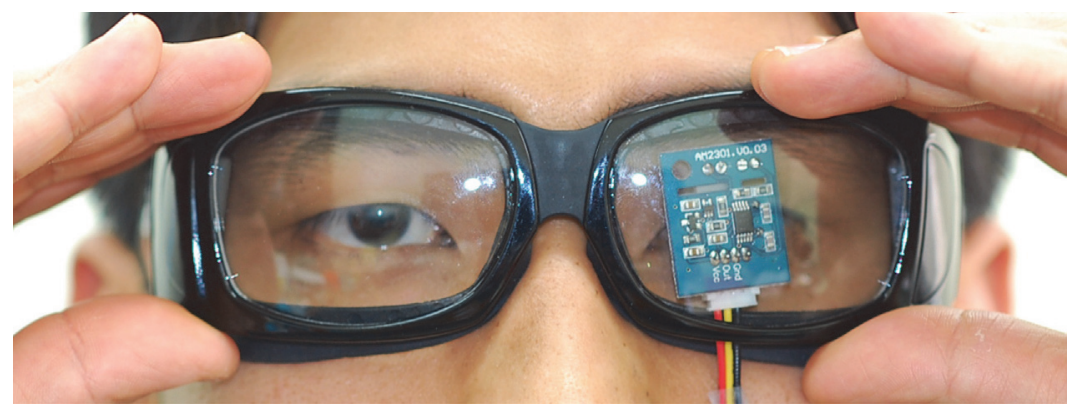

(b)

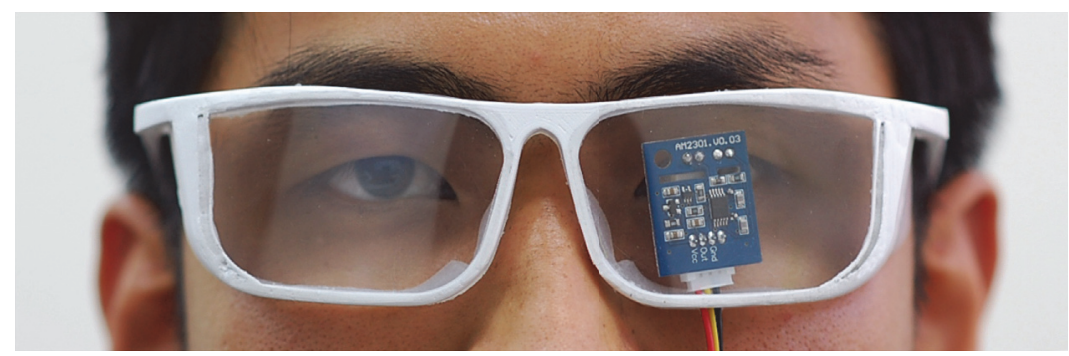

(c)

FIGURE 3: Wearing of moisture chamber spectacles. (a) Natural wearing of uniformed moisture chamber spectacles. (b) Pressurized wearing of uniformed moisture chamber spectacles. (c) Natural wearing of personalized moisture chamber spectacles.

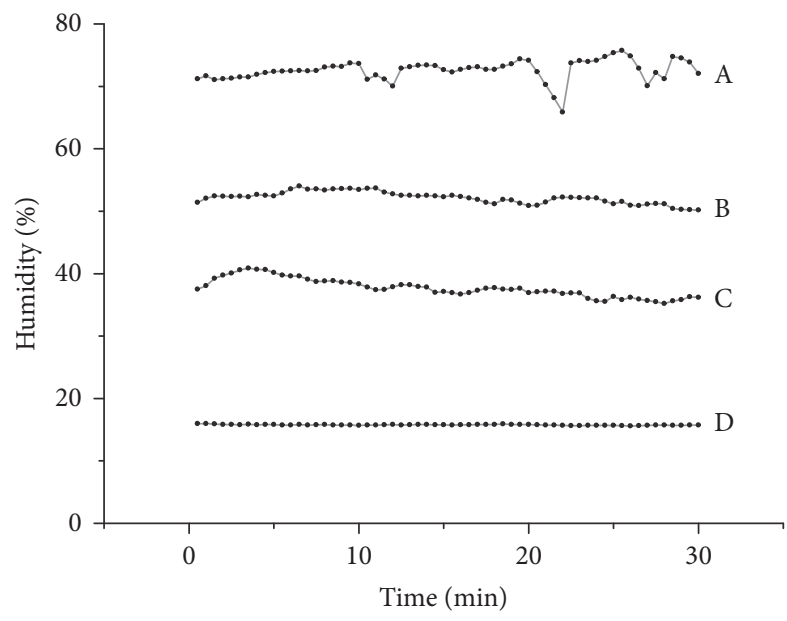

(a)

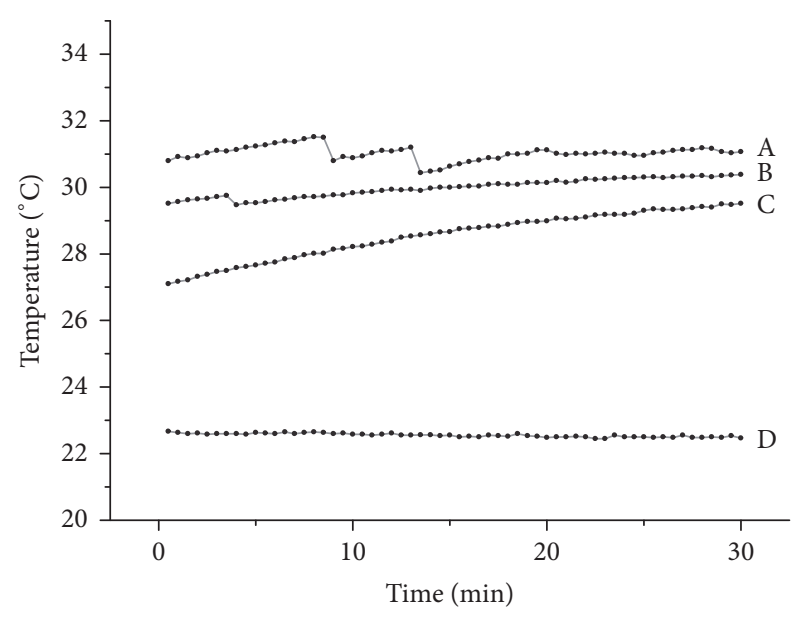

(b)

Figure 4: Measurements of periocular humidity and temperature over time. The average of periocular humidity and temperature over time in 10 volunteers. (a) Pressurized wearing of uniformed moisture chamber spectacles, (b) natural wearing of personalized moisture chamber spectacles, (c) natural wearing of uniformed moisture chamber spectacles, and (d) ambient humidity. 


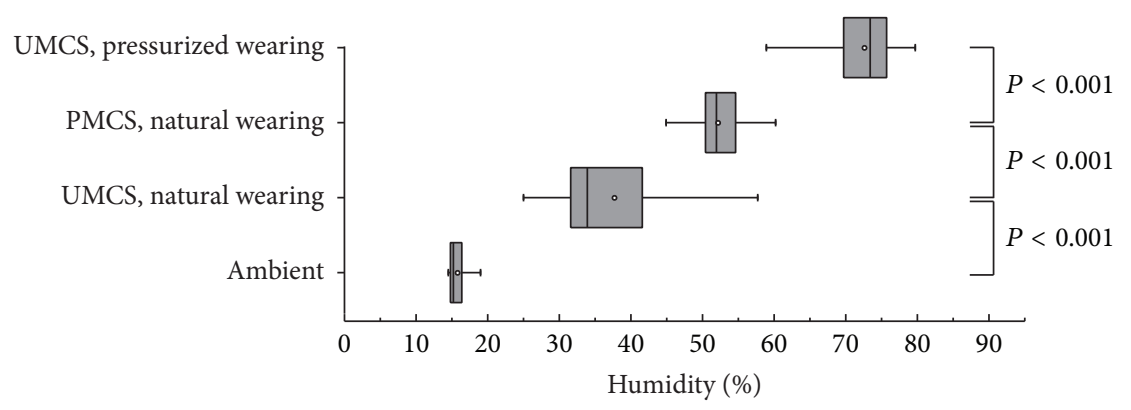

(a)

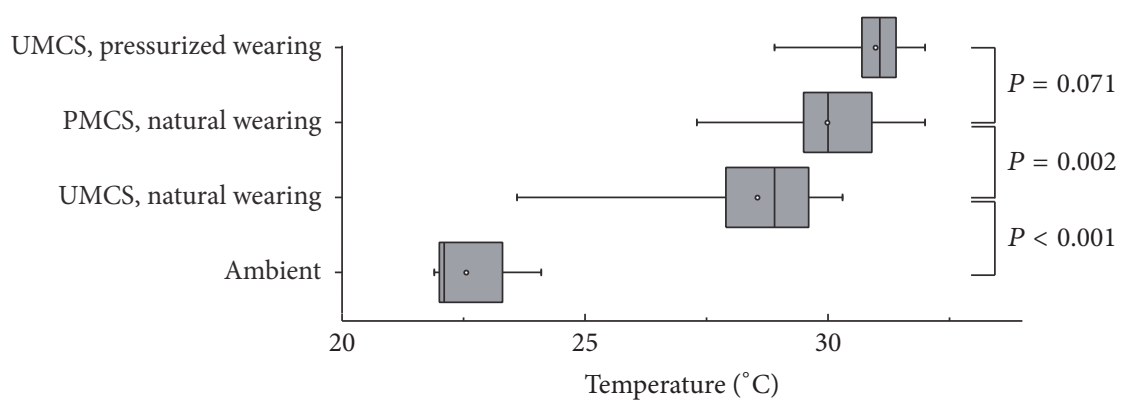

(b)

FIGURE 5: Comparisons of periocular humidity and temperature with various spectacles wearing. Box plots show minimum and maximum (whiskers), 25\% and 75\% quartiles (box), the median (line in the box), and mean (dot in the box).

3.2. Temperature. The thermometer had an accuracy of $\pm 0.5^{\circ} \mathrm{C}$ and a repeatability of $\pm 0.3^{\circ} \mathrm{C}$. Measurements of periocular temperature over time were shown in Figure 4(b). The average ambient temperature was $22.55 \pm 0.76^{\circ} \mathrm{C}$. The mean periocular temperature was highest with pressurized wearing of UMCS $\left(30.98 \pm 0.63^{\circ} \mathrm{C}\right)$, followed by natural wearing of PMCS $\left(29.98 \pm 1.21^{\circ} \mathrm{C}\right)$ and natural wearing of UMCS $\left(28.55 \pm 1.38^{\circ} \mathrm{C}\right)$. Periocular temperature with natural wearing of PMCS was significantly higher than natural wearing of UMCS $(P=0.002)$. However, the difference of periocular temperature between pressurized wearing of UMCS and natural wearing of PMCS was not significant $(P=0.071)$, (Figure 5(b)).

3.3. Condensation. Measured periocular humidity and calculated humidity level that starts to cause the condensation on the spectacles were shown in Figure 6. When periocular humidity becomes greater than calculated condensation humidity, it is indicative of condensation. In this study, measured periocular humidity of natural wearing of UMCS and PMCS demonstrated lower value than calculated condensation humidity at all time points; furthermore, no participants complained of blurred vision due to condensation in practice. However, measured periocular humidity of pressurized wearing of UMCS showed higher value than calculated condensation humidity at all time, and all subjects experienced visual blur.

\section{Discussion}

The wearing of moisture chamber spectacles can be helpful to improve ocular discomfort associated with dry eye. Based on the definition from the dry eye workshop (DEWS), dry eye disease is defined as multifactorial disease that is accompanied by increased osmolarity of the tear film and inflammation of the ocular surface [11]. The DEWS established increased tear osmolarity and tear film instability, followed by goblet cell, glycocalyx mucin loss, and epithelial damage as core mechanisms of dry eye. High evaporation is one of the major causes of tear hyperosmolarity [11]. Tear evaporation rate depends on mass transfer through both the coating lipid layer and ambient air [12]. McCulley et al. reported that 10\% decrease in relative humidity resulted in $28.33 \%$ to $59.42 \%$ increase in tear evaporation [13]. Uchiyama et al. showed that a decrease of relative humidity from $40-45 \%$ to $20-$ $25 \%$ resulted in $99.72 \%$ increase in tear evaporation rate [14]. Additionally, short tear breakup time dry eye is known to be a major part of visual display terminals- (VDT-) associated dry eye [15]. It developed in $66 \%$ of VDT workers and has become increasingly common [16]. Environmental factors such as air drafts and low humidity in the air are known to progressively increase tear water evaporation and promote faster thinning of the precorneal tear film and the consequent ocular surface damage [17].

In this study, PMCS increased absolute value of 36.39\% periocular humidity, as compared to the ambient humidity. Moisture chamber spectacles may be particularly helpful to reduce artificial tear use for severe dry eye, involving Sjögren's syndrome or lagophthalmos patients, and ultra-low humidity environmental worker including laboratory employee [18] or aircraft cabin crew [19].

Appropriate fit is the most critical aspect in moisture chamber spectacles. The loose fitness of spectacles reduces 


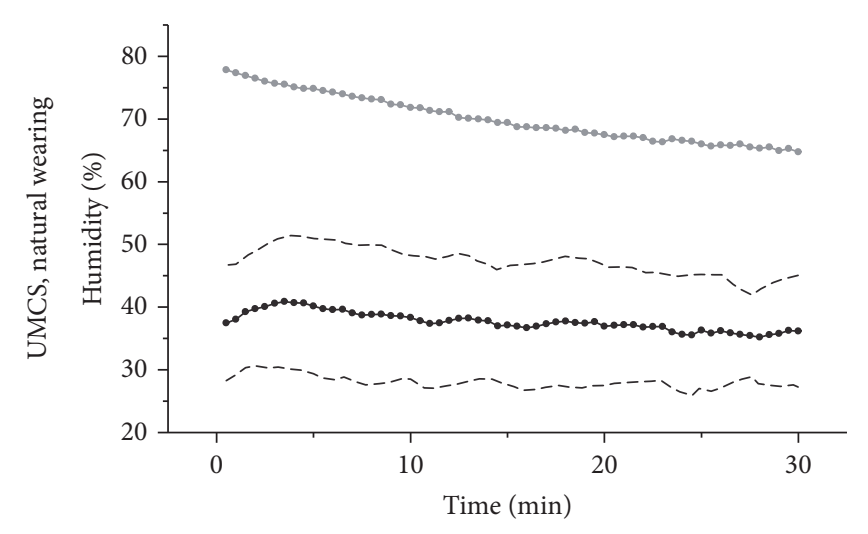

(a)

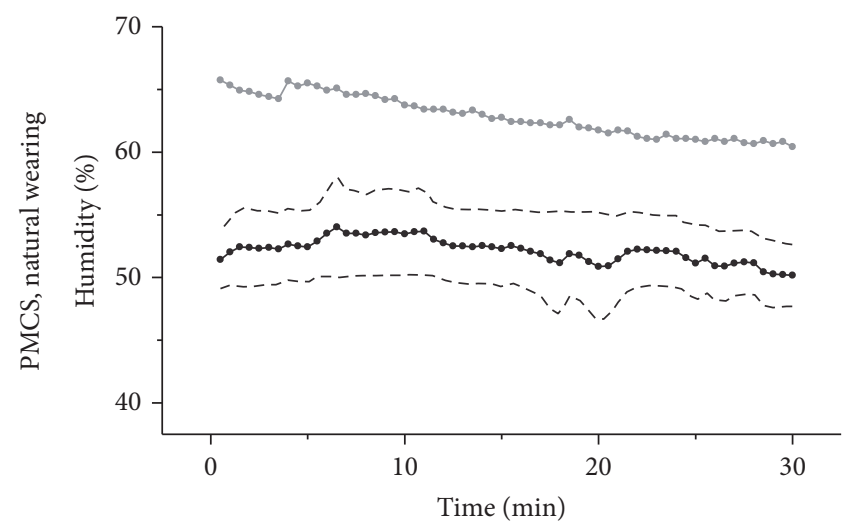

(b)

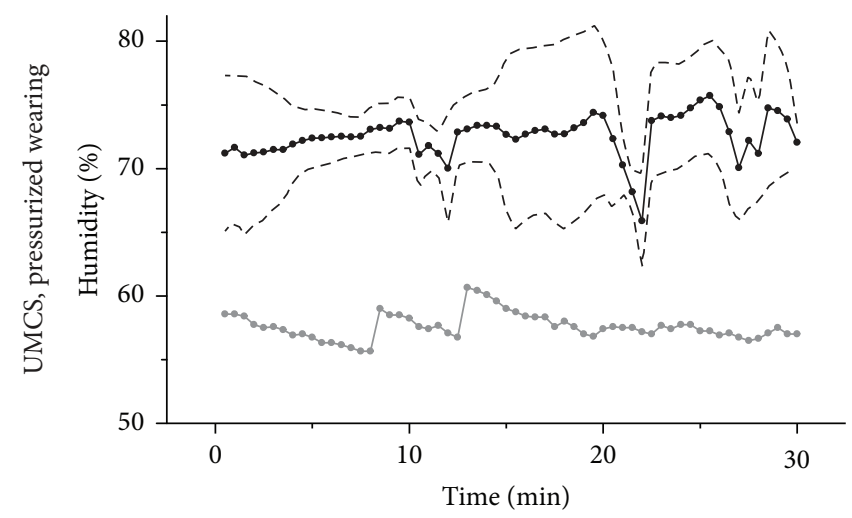

(c)

FIGURE 6: Measured periocular humidity and calculated condensation humidity. Measured periocular humidity (black line), \pm 1 standard deviation of humidity measurements (dash line), and calculated condensation humidity (gray line).

relative humidity in the moisture chamber. In the present study, PMCS showed significantly higher periocular humidity than UMCS, as predicted. Additionally, UMCS demonstrated wide individual differences of periocular humidity (standard deviation $=8.96$ ), while PMCS showed narrow individual variations (standard deviation $=3.00$ ). In contrast, tight fitness of spectacles induces excessively high relative humidity, resulting in condensation as well as discomfort. Moisture chamber should be a semiclosed, and not a completely closed space due to condensation. In this study, pressurized wearing of UMCS which formed near completely closed space showed higher mean periocular humidity than condensation humidity. Though the higher periocular humidity provides greater tear retention effect, the spectacles inducing condensation without antifog material are not practical for daily wear. PMCS provided sufficiently high periocular humidity; however the measurements were always slightly lower than the condensation point. These results suggested that personalization of moisture chamber provides appropriate fit.

There were some limitations to this study. The present study had small sample size and did not include patient group and clinical evaluation. Further investigation will be needed to determine the clinical effectiveness of PMCS, involving subjective symptoms and objective ocular surface evaluation in patients with dry eye disease with larger sample size.

\section{Conclusions}

PMCS made by 3D printer provides appropriate fitness to form semiclosed humid chamber. PMCS increased absolute value of $36.39 \%$ periocular humidity without condensation as compared to the ambient humidity and demonstrated significantly higher performance than UMCS. The wearing of PMCS would be an effective method to provide highly enough periocular humidity in low humidity environment.

\section{Ethical Approval}

All procedures followed were in accordance with the ethical standards of the responsible committee on human experimentation (institutional and national) and with the Helsinki Declaration of 1964, as revised in 2013.

\section{Consent}

Informed consent was obtained from all patients for being included in the study. 


\section{Disclosure}

The opinions expressed herein are that of the authors and do not reflect the official policy or position of the Republic of Korea Air Force or Republic of Korea Ministry of National Defense.

\section{Competing Interests}

The authors declare that they have no competing interests.

\section{Acknowledgments}

This work was supported by a grant of the Aerospace Medicine Research Project funded by the Medical Division, Headquarter, Republic of Korea Air Force (ROKAF-ASMC2015-AMRP-001), and partially by the Soonchunhyang University Research Fund.

\section{References}

[1] M. Hessen and E. K. Akpek, "Dry eye: an inflammatory ocular disease," Journal of Ophthalmic and Vision Research, vol. 9, no. 2, pp. 240-250, 2014.

[2] D. R. Korb, J. V. Greiner, T. Glonek, R. Esbah, V. M. Finnemore, and A. C. Whalen, "Effect of periocular humidity on the tear film lipid layer," Cornea, vol. 15, no. 2, pp. 129-134, 1996.

[3] K. Tsubota, M. Yamada, and K. Urayama, "Spectacle side panels and moist inserts for the treatment of dry-eye patients," Cornea, vol. 13, no. 3, pp. 197-201, 1994.

[4] D. A. Zopf, S. J. Hollister, M. E. Nelson, R. G. Ohye, and G. E. Green, "Bioresorbable airway splint created with a threedimensional printer," The New England Journal of Medicine, vol. 368, no. 21, pp. 2043-2045, 2013.

[5] M. S. Mannoor, Z. Jiang, T. James et al., "3D printed bionic ears," Nano Letters, vol. 13, no. 6, pp. 2634-2639, 2013.

[6] B. Leukers, H. Gülkan, S. H. Irsen et al., "Hydroxyapatite scaffolds for bone tissue engineering made by 3D printing," Journal of Materials Science: Materials in Medicine, vol. 16, no. 12, pp. 1121-1124, 2005.

[7] W. Huang and X. Zhang, "3D printing: print the future of ophthalmology," Investigative Ophthalmology and Visual Science, vol. 55, no. 8, pp. 5380-5381, 2014.

[8] R. L. Scawn, A. Foster, B. W. Lee, D. O. Kikkawa, and B. S. Korn, "Customised 3D printing: an innovative training tool for the next generation of orbital surgeons," Orbit, vol. 34, no. 4, pp. 216-219, 2015.

[9] A. Ruzza, M. Parekh, S. Ferrari et al., "Preloaded donor corneal lenticules in a new validated 3D printed smart storage glide for Descemet stripping automated endothelial keratoplasty," British Journal of Ophthalmology, vol. 99, no. 10, pp. 1388-1395, 2015.

[10] M. G. Lawrence, “The relationship between relative humidity and the dewpoint temperature in moist air: a simple conversion and applications," Bulletin of the American Meteorological Society, vol. 86, no. 2, pp. 225-233, 2005.

[11] "The definition and classification of dry eye disease: report of the Definition and Classification Subcommittee of the International Dry Eye WorkShop," The Ocular Surface, vol. 5, pp. 75-92, 2007.
[12] C.-C. Peng, C. Cerretani, R. J. Braun, and C. J. Radke, "Evaporation-driven instability of the precorneal tear film," Advances in Colloid and Interface Science, vol. 206, pp. 250-264, 2014.

[13] J. P. McCulley, J. D. Aronowicz, E. Uchiyama, W. E. Shine, and I. A. Butovich, "Correlations in a change in aqueous tear evaporation with a change in relative humidity and the impact," American Journal of Ophthalmology, vol. 141, no. 4, pp. 758-760, 2006.

[14] E. Uchiyama, J. D. Aronowicz, I. A. Butovich, and J. P. McCulley, "Increased evaporative rates in laboratory testing conditions simulating airplane cabin relative humidity: an important factor for dry eye syndrome," Eye and Contact Lens, vol. 33, no. 4, pp. 174-176, 2007.

[15] M. Kaido, M. Kawashima, N. Yokoi et al., "Advanced dry eye screening for visual display terminal workers using functional visual acuity measurement: the Moriguchi study," British Journal of Ophthalmology, vol. 99, no. 11, pp. 1488-1492, 2015.

[16] M. Uchino, N. Yokoi, Y. Uchino et al., "Prevalence of dry eye disease and its risk factors in visual display terminal users: the Osaka study," American Journal of Ophthalmology, vol. 156, no. 4, pp. 759-766.e1, 2013.

[17] M. Hirayama, D. Murat, Y. Liu, T. Kojima, T. Kawakita, and K. Tsubota, "Efficacy of a novel moist cool air device in office workers with dry eye disease," Acta Ophthalmologica, vol. 91, no. 8, pp. 756-762, 2013.

[18] M. Sato, S. Fukayo, and E. Yano, "Adverse environmental health effects of ultra-low relative humidity indoor air," Journal of Occupational Health, vol. 45, no. 2, pp. 133-136, 2003.

[19] N. L. Nagda, "Low relative humidity and aircraft cabin air quality," Indoor Air, vol. 11, no. 3, pp. 200-214, 2001. 


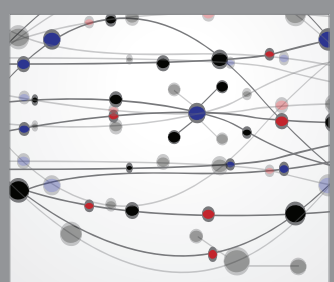

The Scientific World Journal
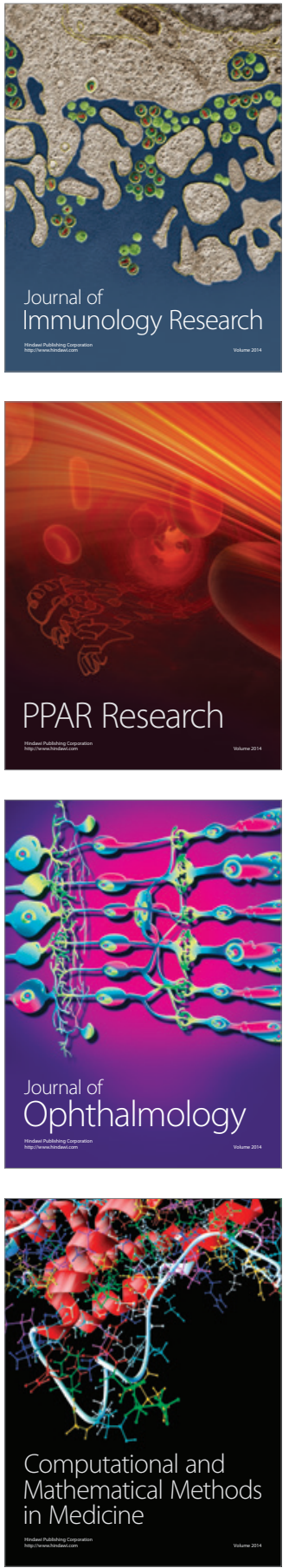

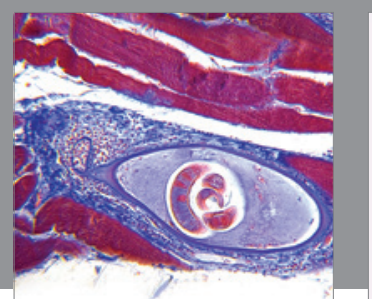

Gastroenterology Research and Practice

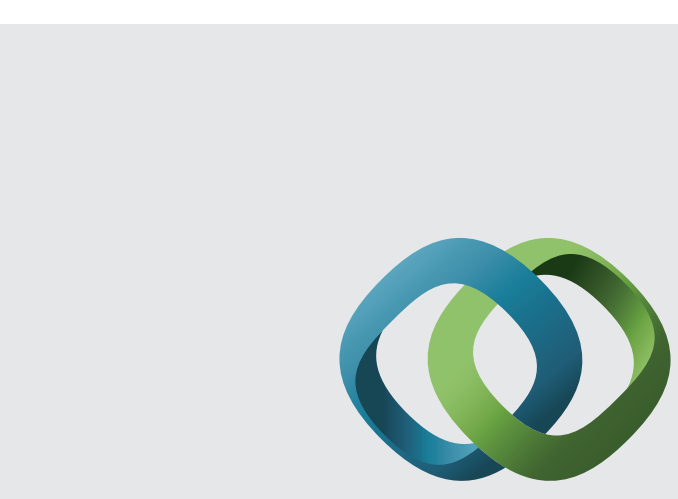

\section{Hindawi}

Submit your manuscripts at

http://www.hindawi.com
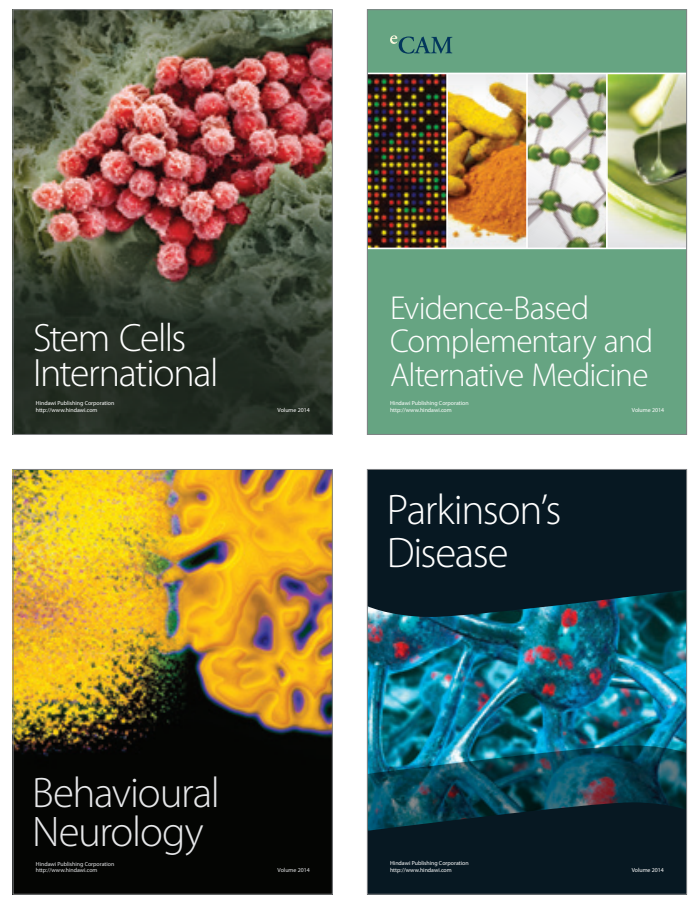
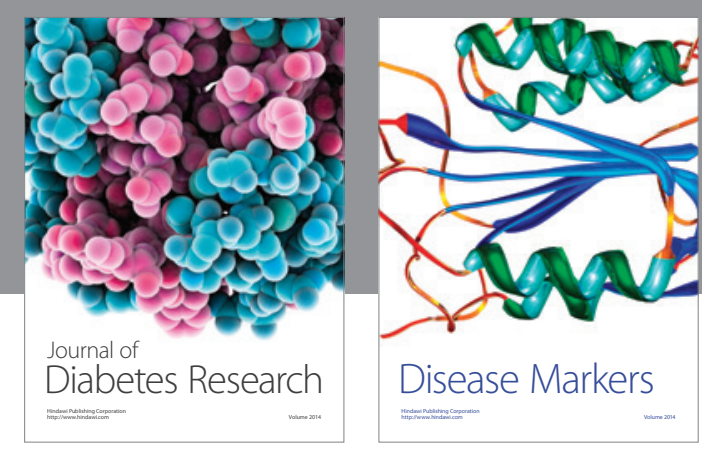

Disease Markers
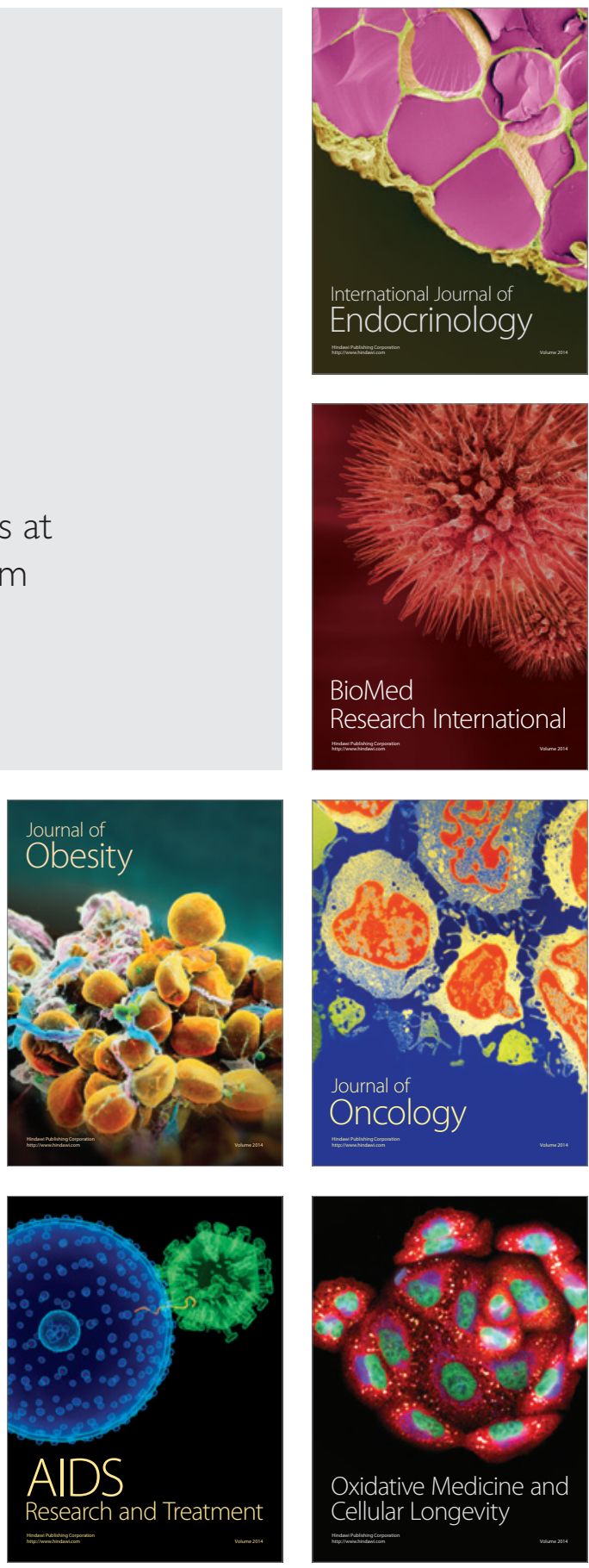